\title{
EXPERIMENTAL STUDY OF MINUTIAE BASED ALGORITHM FOR FINGERPRINT MATCHING
}

\author{
Cleopas Officer Angaye ${ }^{1}$, Oluwole Charles Akinyokun ${ }^{2}$ and \\ Gabriel Babatunde Iwasokun ${ }^{2}$ \\ ${ }^{1}$ National Information Technology Development Agency (NITDA), \\ Abuja, Nigeria \\ ${ }^{2}$ Department of Computer Science, \\ Federal University of Technology, Akure, Nigeria \\ cangaye@hotmail.com, charliebobo2012@yahoo.co.uk, \\ maxtunde@yahoo.com
}

\begin{abstract}
In this paper, a minutiae-based algorithm for fingerprint pattern recognition and matching is proposed. The algorithm uses the distance between the minutiae and core points to determine the pattern matching scores for fingerprint images. Experiments were conducted using FVC2002 fingerprint database comprising four datasets of images of different sources and qualities. False Match Rate (FMR), False Non-Match Rate (FNMR) and the Average Matching Time (AMT) were the statistics generated for testing and measuring the performance of the proposed algorithm. The comparative analysis of the proposed algorithm and some existing minutiae based algorithms was carried out as well. The findings from the experimental study were presented, interpreted and some conclusions were drawn.
\end{abstract}

\section{KEYWORDS}

Minutiae, Segmentation, Normalization, Gabor Filter, Binarization, Bifurcation

\section{INTRODUCTION}

The issue of identity management of individuals, organizations and other public and private institutions poses a great challenge to government worldwide today. Biometric identification has featured prominently for individuals with fingerprint emerging as the dominant one. The dominance of fingerprint is been established by the continuous emergence of different forms of Automated Fingerprint Identification Systems (AFIS). In the course of performing its assigned roles, an AFIS conducts a lot of activities including fingerprint enrolment and creation of its profile database, minutiae detection (image segmentation, normalization, Gabor filter, binarization/thining), extraction and database creation, pattern recognition, pattern matching, error detection and correction, and decision making. Fingerprint is an impression of the friction ridges of all or any part of the finger formed when a finger touches a surface. The ridges are represented by raised and dark portions while the valleys are the white and lowered regions as

David C. Wyld (Eds) : ICCSEA, SPPR, CSIA, WimoA - 2013

pp. 33-47, 2013. @ CS \& IT-CSCP 2013

DOI : 10.5121/csit.2013.3504 
shown in Figure 1. Facts exist that the ridges of individual finger never change throughout his or her lifetime no matter what happens. Even in case of injury or mutilation, they will always reappear within a short period. Five commonly found fingerprint patterns are arch, tented arch, left loop, right loop and whorl as presented in Figure 2.

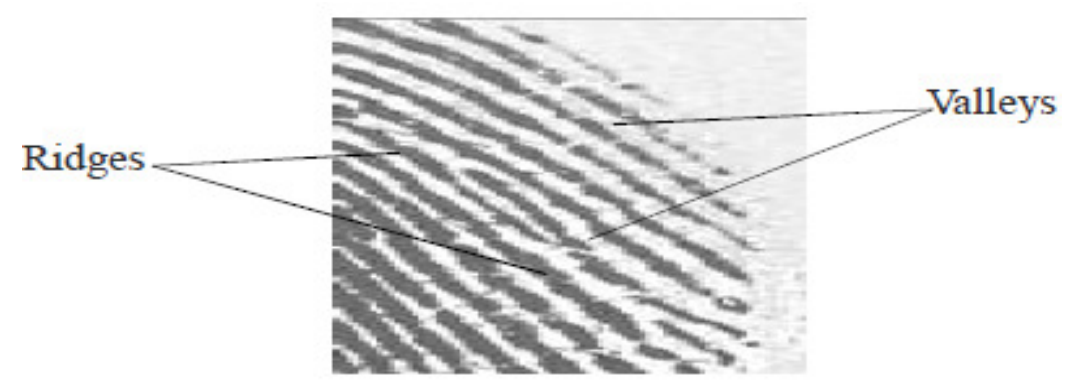

Figure 1: Fingerprint Ridge and Valley

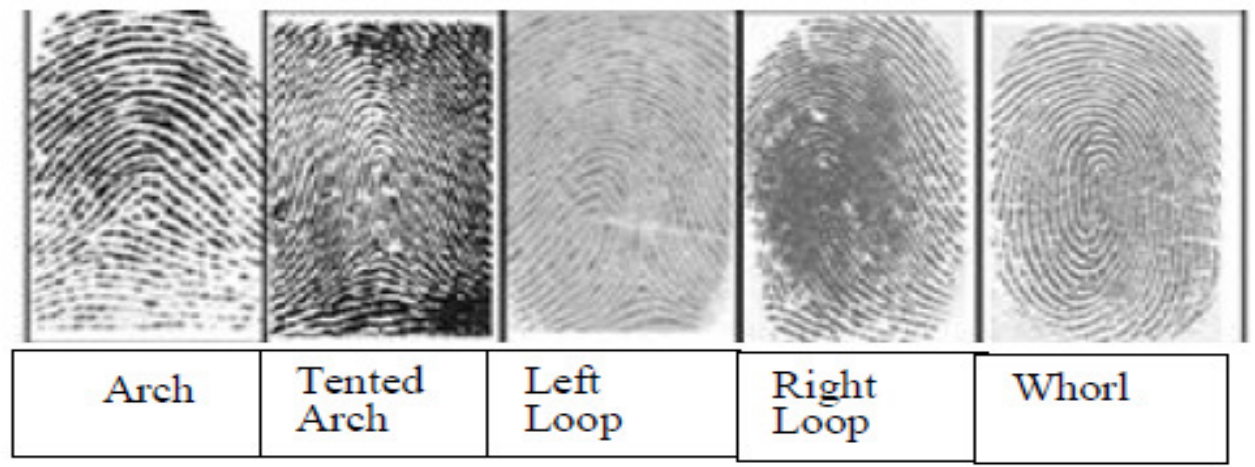

Figure 2: Common Fingerprint Patterns

The American Biometrics Groups in their recent survey [1], adjudged fingerprint as the most reliable human identification and verification index with superiority over all other biometrics including ear, nose, iris, voice, face, gait and signature. The result of the survey is presented in Figure 3.

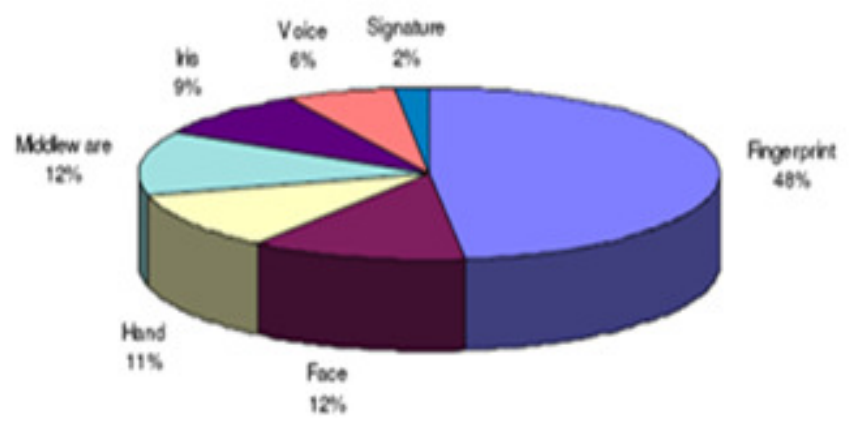

Figure 3: Statistics of Biometrics Identification 
The mostly used fingerprint components in identification and authentication include ridge bifurcations and endings as shown in Figure 4. The minutiae points are the points that uniquely describe any fingerprint image. A minutia point is described by type, location and orientation. Algorithms for the extraction of minutiae points from thinned fingerprint images have been proposed in [2-5].

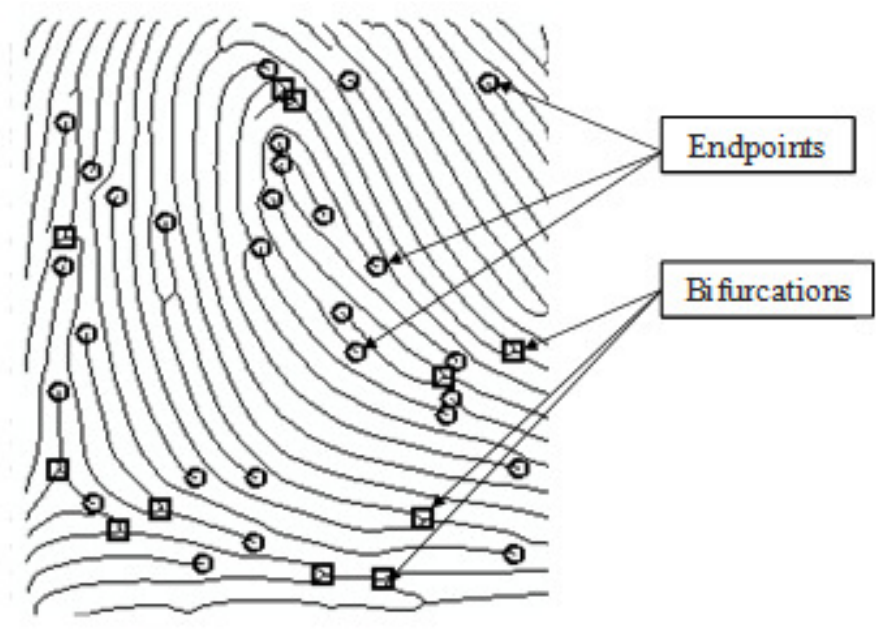

Figure 4: Fingerprint Bifurcation and Endpoints

The template size of the biometric information base on minutiae and computational time is relatively low compared with that of other techniques such as graph-based fingerprint matching [6]. During minutiae-based fingerprint pattern matching, a match score between two fingerprints is computed based on the characteristics exhibited by the minutiae. The basic idea in several minutiae based techniques is connecting the neighbor minutiae with triangles using a Delaunay triangulation and analyzing the relative position and orientation of the grouped minutiae [7-8]. While that technique provides a good processing time, describes the minutia relationship with consistency and works well with the nonlinear distortions, for genuine match, the overlapping area between the matching fingerprints should be large.

Limitations of existing minutiae based fingerprint pattern recognition and matching algorithms are:

a. Large intra-class variations (variations in fingerprint images of the same finger) and large inter-class similarity (similarity between fingerprint images from different fingers) [9].

b. Slow in response due to series of non-optimized procedures [10-11].

c. Performance diminishes with images with high number of minutiae [11].

d. Failure in cases of poor quality and noisy images [10, 12].

e. Collapse when ridge map not available [13].

f. fIrregular similarity scores [14]. 


\section{IMPROVEd MinUtiae BASEd FingerPRINT PATTERn MATCHING ALGORITHM}

The approach in [7-8] is similar to the approach taken in the study and reported in this paper. However, we proposed a new method for generating fingerprints matching scores using the spatial parameters existing between the minutiae points. The method proved suitable enough for handling matching problems due to image ridge orientation and size variations. The proposed algorithm for the computation of the pattern matching scores for fingerprint images relies on the distances between the image core and the minutiae points. The formulation of the algorithm was motivated by the fact that the relative distance to the core point from each minutia point does not change irrespective of the image directional flow. The core point being the point of maximum turning is the point at which the gradient is zero. The core points A and B shown in Figure 5 are the points of maximum turning of the ridge structures in the two images. They are also the points where the directional fields experience total orientation changes [15-16].

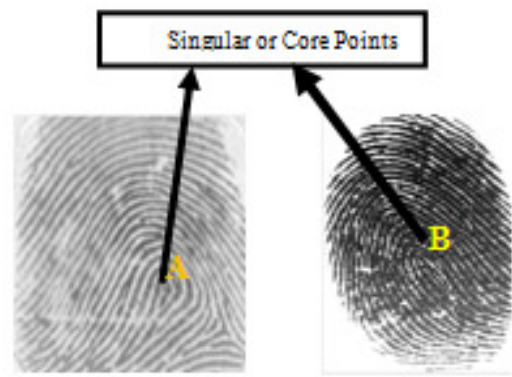

Figure 5: Fingerprint images and their core

The importance of these points is that their positions do not change and constant distance is always maintained between them and every feature point irrespective of direction for a specific image size. Among the common feature points that uniquely describe a fingerprint image are bifurcations and ridge endings (shown in Figure 4) [3,5]. A circle and square in Figure 6(a) and Figure 6(b) represent bifurcation and ridge ending respectively. The thick diamonds represent the core points.

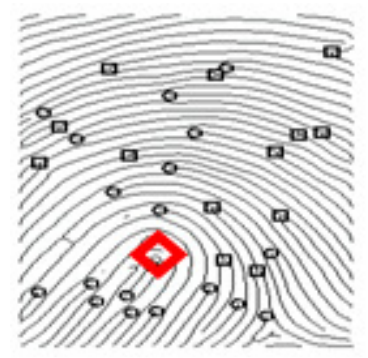

(a) Skeleton image

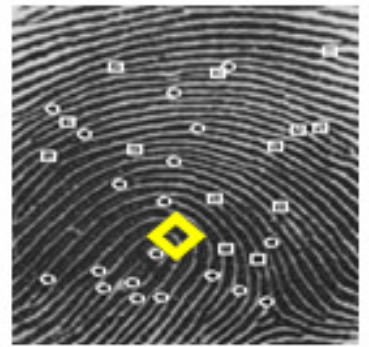

(b) Original image

Figure 6: Feature points for skeleton and original images

Figure 7 illustrates typical interconnecting lines between nine (9) minutiae points labeled A, B, C, $\mathrm{D}, \mathrm{E}, \mathrm{F}, \mathrm{G}, \mathrm{H}, \mathrm{I}$ and the core point $\mathrm{O}$ in the enclosed region of an image. The connecting lines are in different directions with lengths depending on the distances apart between point $\mathrm{O}$ and the connecting minutiae points. 


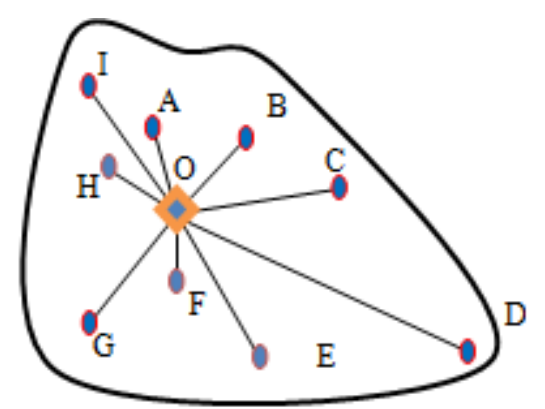

Figure 7: Interconnecting lines between feature and core points

The procedure for the proposed algorithm is in the following phases:

a. Obtain the core point using the following procedure of the algorithm presented in [17].

- Divide the fingerprint image, I, into blocks of size $\mathrm{N} \mathrm{x} \mathrm{N}$.

- Compute the orientation estimate for the center pixel $A(i, j)$ of each block.

- Compute the sine component in radian of each estimate using $\sin (A(\mathrm{i}, \mathrm{j}))$

A perfectly horizontal ridge has a sine component of 0 while vertical ridge has a sin component of 1 . Due to the discontinuity property, the sine component value always changes abruptly from 0 to 1 or vice versa at the core point. In view of this, the following additional operations are performed:

- Initialize a 2 dimensional array $\mathrm{B}_{\mathrm{i}}(\mathrm{i}, \mathrm{j})$ and set all its entries to 0 .

- Scan the sine components map in a top-to-bottom, left-to-right manner. For each sine component

$$
\beta(i, j)=\operatorname{Sine}(A(i, j)),
$$

If $\beta(\mathrm{i}, \mathrm{j})<A$ threshold and $\beta(\mathrm{i}-1, \mathrm{j})>\mathrm{p} / 2$ and $\beta(\mathrm{i}+1, \mathrm{j})>\mathrm{p} / 2$ then

Compute the difference D.

Compute the $\mathrm{B}_{\mathrm{i}}(\mathrm{i}, \mathrm{j})$

End if

The difference $\mathrm{D}$ between the sine components for block with center at pixel $(\mathrm{i}, \mathrm{j})$ and another block with center pixel at $(\mathrm{k}, \mathrm{l})$ is denoted by:

$$
\mathrm{D}=\operatorname{Sin}(\mathrm{i}, \mathrm{j})-\operatorname{Sin}(\mathrm{k}, \mathrm{l})
$$

The $\mathrm{B}(\mathrm{i}, \mathrm{j})$ entry is used to compute the continuity of a possible reference candidate point and is defined as:

$$
B(i, j)=\left\{\begin{array}{c}
1 ; \quad \text { if } \mathrm{i}==1 \\
\operatorname{Bi}(\mathrm{i}-1, \mathrm{j}-1)+\operatorname{Bi}(\mathrm{i}-1, \mathrm{j})+\operatorname{Bi}(\mathrm{i}-1, \mathrm{j}+1) ; \text { otherwise }
\end{array}\right.
$$

b.Obtain the $\mathrm{x}$ and $\mathrm{y}$ coordinates for all the true bifurcations and ridge endings in the thinned image. The Crossing Number $(\mathrm{CN})$ value for a candidate ridge ending and bifurcation is obtained according to the formula: 


$$
C N=\sum_{i=0}^{7}\left|N_{i+2}-N_{i+1}\right|, \quad N_{9}=N_{1}
$$

$\mathrm{N}_{1}, \mathrm{~N}_{2}, \ldots, \mathrm{N}_{8}$ denote the 8 neighbours of the candidate minutia point contained in its $3 \times 3$ neigbourhood. The 8neigbours of a candidate pixel $\mathrm{N}$ are scanned in clockwise direction as follows:

\begin{tabular}{|l|l|l|}
\hline $\mathrm{N}_{2}$ & $\mathrm{~N}_{3}$ & $\mathrm{~N}_{4}$ \\
\hline $\mathrm{N}_{1}$ & $\mathrm{~N}$ & $\mathrm{~N}_{5}$ \\
\hline $\mathrm{N}_{8}$ & $\mathrm{~N}_{7}$ & $\mathrm{~N}_{6}$ \\
\hline
\end{tabular}

As shown in Figure 8, a ridge pixel with $\mathrm{CN}$ value of 2 corresponds to a ridge ending and a $\mathrm{CN}$ value of 6 corresponds to a bifurcation.

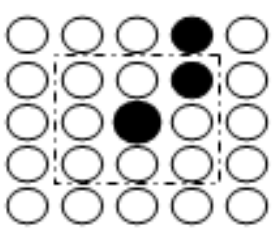

(a) $\mathrm{CN}=2$

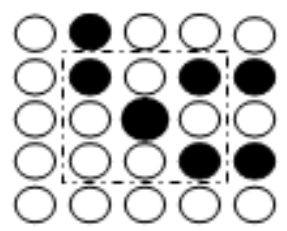

(b) $\mathrm{CN}=6$

Figure 8: $\mathrm{CN}$ values for ridge ending and bifurcation points

To ensure that only valid minutiae are extracted from the image, a minutiae validation algorithm proposed in [5] is implemented. The algorithm tests the validity of each candidate minutiae point by scanning the skeleton image and examines the local neighbourhood around the point. Firstly, an image $M$ of size $W x W$ centered on the candidate minutiae point in the skeleton image is created. Secondly, the central pixel of $M$ is labelled with a value of 2 and the rest of the pixels in $M$ are initialised to values of zero. The subsequent steps of the algorithm depend on whether the candidate minutiae point is a ridge ending or a bifurcation.

For a candidate bifurcation point:

- Examine the $3 \times 3$ neighbourhood of the bifurcation point in a clockwise direction. For the three pixels that are connected with the bifurcation point, label them with the value of 1.

- Label with 1 the three ridge pixels that are connected to these three connected pixels.

- Count in a clockwise direction, the number of transitions from 0 to $1\left(T_{01}\right)$ along the border of image $M$. If $T_{01}=3$, then the candidate minutiae point is validated as a true bifurcation

For a candidate ridge ending point:

- Label with a value of 1 all the pixels in $M$, which are in the $3 \times 3$ neighbourhood of the ridge ending point.

- Count in a clockwise direction, the number of 0 to 1 transitions $\left(T_{01}\right)$ along the border of image $M$. If $T_{01}=1$, then the candidate minutiae point is validated as a true ridge ending. 
c. The distance, $\lambda_{\mathrm{i}}$ between the $\mathrm{i}^{\text {th }}$ minutiae point $\mathrm{P}_{\mathrm{i}}\left(\mathrm{a}_{\mathrm{i}}, \mathrm{b}_{\mathrm{i}}\right)$ and the image core point $\mathrm{M}(\rho, \sigma)$ is obtained from:

$$
\lambda_{\mathrm{i}}=\left(\left(a_{i}-\rho\right)^{2}+\left(b_{i}-\sigma\right)^{2}\right)^{0.5}
$$

d.The degree of closeness $E_{c}$ is obtained for matching image $\mathrm{K}$ with image $\mathrm{L}$ by using the formula:

$$
E_{c}=\sum_{i=1}^{s}(|G(i)-H(i)|) *\{G(i)\}^{-1}
$$

$\mathrm{s}$ is the smaller of the respective number of feature points in the two images, G(i) and $\mathrm{H}(\mathrm{i})$ represent the distance between the $\mathrm{i}^{\text {th }}$ minutiae point and the core point in $\mathrm{K}$ and $\mathrm{L}$ respectively.

e. The correlation coefficient value, $\mathrm{S}$ between $\mathrm{K}$ and $\mathrm{L}$, is then computed as the pattern matching score by using the formula:

$$
S=\left(1-E_{c}\right) * 10^{-2}
$$

From this formula, the closeness value will be $E_{c}=0$ for exact or same images and, consequently, the correlation will be $\mathrm{S}=1$.

\section{EXPERIMENTAL STUDY}

The implementation of the proposed fingerprint matching algorithm was carried out using Matlab version 7.6 on Ms-Window Vista Home Basic Operating System. The experiments were performed on a Pentium $4-2.10 \mathrm{GHz}$ processor with $1.00 \mathrm{~GB}$ of RAM. The experiments were conducted for the analysis of the performance of the proposed algorithm when subjected to images with various conditions. The experiments also serve the basis for the generation of metric values that are relevant in the comparison of the results from the research with results from related works. The case study of fingerprint images obtained from Fingerprint Verification Competition was carried out. The fingerprints are contained in FVC2002fingerprint database with datasets DB1, DB2, DB3 and DB4 [18]. A summary of the database is presented in Table 1.

The database contains benchmark fingerprints jointly produced by The Biometric Systems Laboratory, Bologna, Pattern Recognition and Image Processing Laboratory, Michigan and the

\begin{tabular}{|c|c|c|c|c|}
\hline Data-base & Sensor Type & Image size & Number & Resolution \\
\hline DB1 & Optical Sensor & $\begin{array}{l}388 \times 374(142 \\
\text { Kpixels })\end{array}$ & $100 \times 8$ & $500 \mathrm{dpi}$ \\
\hline DB2 & $\begin{array}{l}\text { Optical } \\
\text { Sensor }\end{array}$ & $\begin{array}{l}296 \times 560(162 \\
\text { Kpixels })\end{array}$ & $100 \times 8$ & 569 dpi \\
\hline DB3 & $\begin{array}{l}\text { Capacitive } \\
\text { Sensor }\end{array}$ & $\begin{array}{l}300 \times 300(88 \\
\text { Kpixels })\end{array}$ & $100 \times 8$ & 500 dpi \\
\hline DB4 & SFinGe v2.51 & $\begin{array}{l}288 \times 384(108 \\
\text { Kpixels })\end{array}$ & $100 \times 8$ & $\begin{array}{l}\text { About } \\
500 \text { dpi }\end{array}$ \\
\hline
\end{tabular}
Biometric Test Center, San Jose, United States of America. Each of the four datasets contains 80

Table 1: Details of FVC2002 fingerprint database 
images that differ in qualities. The 80 fingerprints are made up of 10 fingerprints from 8 different fingers and persons. The first two datasets were acquired using an optical fingerprint reader. The third and fourth datasets were acquired using capacitive fingerprint reader and computer software assistance respectively.

False non-match rate (FNMR), false match rate (FMR) and average matching time(AMT) were the indicators that were measured. Matching all the fingerprints from the same finger or person was used to measure the FNMR while measuring FMR is done through matching each fingerprint image of each of the eight persons with all other fingerprints from the other persons. The obtained results revealed the factors affecting the indicators. For instance, facts emerged that false nonmatch rate and false match rate results are greatly affected by the nature and quality of the images. The FMR and FNMR results obtained for a threshold value for the first two datasets are shown in Table 2 and Table 3 respectively.

Table 2: FMR and FNMR Values for Dataset DB1

\begin{tabular}{cc}
\hline Statistics & Value $(\%)$ \\
\hline FMR & 0 \\
FNMR & 22.23 \\
\hline
\end{tabular}

The results from Table 2 and Table 3 reveal that for images obtained using optical fingerprint reader, the proposed algorithm produced an FMR of $0 \%$. Meaning that the algorithm did excellently in identifying from the two datasets, fingerprint images obtained from different fingers under equal conditions. However, the obtained FNMR values of $22.23 \%$ and $19.85 \%$ show the extent to which the algorithm failed to match reasonable number of fingerprint images in the two datasets even though concerned images were obtained from the same person. Some factors may be responsible for this. They include variation in pressure, rotation, translation and contact area during enrolment [9]. These factors constrained images from the same finger to show difference in quality, contrast and noise levels. Consequently, different matching scores are obtained for different pairs. The obtained FNMR values indicate the extent to which these factors adversely affect the images in each dataset.

Table 3: FMR and FNMR Values for Dataset DB2

\begin{tabular}{cc}
\hline Statistics & Value (\%) \\
\hline FMR & 0 \\
FNMR & 19.85 \\
\hline
\end{tabular}

The obtained FMR and FNMR values obtained for the third dataset are presented in Table 4. The results show that for the images in the datasets, the proposed algorithm produced an FMR of $0 \%$. This shows a perfect performance level for the algorithm in the identification and separation of fingerprint images captured from different fingers using capacitive fingerprint reader under same conditions. However, the obtained FNMR value of $14.51 \%$ revealed the failure extent of the algorithm in its attempt to match the fingerprint images enrolled from same finger. This low failure rate is attributed to improvement in quality of the images in dataset DB3 over the images in the first two datasets.. Visual inspection of fingerprints images in dataset DB3 reveals significant reduction in sizes and greater clarity which led to better enhancement, extraction of only true minutiae points and matching. The higher FNMR values in the first two datasets mean that the enhancement process is more adversely affected by artifacts. Artifacts are the foreign ridge and valley structure introduced into the image during the enhancement process. They may be inform of cross over, hole or spike structures [5]. Some of these artifacts may mislead the 
validation algorithm into the extraction of false minutiae (ridge ending and bifurcation) points across the images. The effect of these false extractions is the higher FNMR rate. The lowest FNMR value for the third dataset establishes its superiority over the first two datasets in term of quality and reduction in the number of false minutiae extraction.

\begin{tabular}{cc} 
Table 4: FMR and FNMR Values for & Dataset DB3 \\
\hline Statistics & Value $(\%)$ \\
\hline FMR & 0 \\
FNMR & 14.51 \\
\hline
\end{tabular}

Dataset DB4's FMR and FNMR values are shown in Table 5. These values revealed that the proposed algorithm produced an FMR of $0 \%$ for the dataset. The significance of this value is that the algorithm superbly and completely separated fingerprint images captured from different fingers using computer aids. However, the obtained FNMR value of $16.47 \%$ revealed the degree at which the algorithm could not match the concerned images. Visual inspection of the 80 fingerprint images in dataset DB4 reveals better connection between the ridges when compared with images in datasets DB1 and DB2. This is why dataset DB4's FNMR value is lower than what obtained for datasets DB1 and DB2. However, compared with the FNMR value for dataset DB3, the higher FNMR recorded for dataset DB4 indicates that the images in dataset DB3 are better in terms of ridge connections and qualities. This also implies that gaps across the ridges in dataset DB4 show greater adversity and extraction of false minutiae. The recorded FNMR value of $16.47 \%$ therefore indicates that these false minutiae points impacted more negatively in dataset DB4 than in dataset DB3.

Table 5: FMR and FNMR for Dataset DB4

\begin{tabular}{cc}
\hline Statistics & Value $(\%)$ \\
\hline FMR & 0 \\
FNMR & 16.47 \\
\hline
\end{tabular}

The straight-line graph of Figure 9 and the column chart of Figure 10 present the trend of the FNMR values for the four datasets.

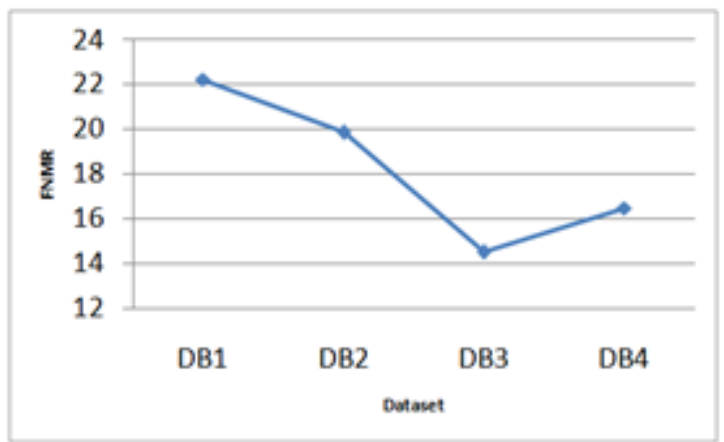

Figure 9: The trend of FNMR values for the four datasets

The two figures show that the FNMR values decreases in the order of 22.23, 19.85, 16.47 and 14.51 for datasets DB1, DB2, DB4 and DB3 respectively. This order proved or established that the images in dataset DB3 is best in terms of quality while dataset DB1 is the worst. In the overall, the proposed pattern matching algorithm successfully separated fingerprints based on source (finger) by returning an average FMR of $0 \%$ for the fingerprints images obtained from different sources and methodologies in the four datasets. From the FNMR value, an average 
FNMR value of $18.26 \%$ is recorded as the failure rate of the proposed algorithm over the four datasets. The average matching times for FNMR and FMR in the four datasets are presented in Table 6.

Table 6: Average Matching Time for Four Datasets

\begin{tabular}{ccc}
\hline Dataset & \multicolumn{2}{c}{ Average Matching time (sec) } \\
& FNMR & FMR \\
\hline DB1 & 1.16 & 1.61 \\
DB2 & 0.91 & 1.27 \\
DB3 & 0.79 & 0.93 \\
DB4 & 0.86 & 0.89 \\
\hline
\end{tabular}

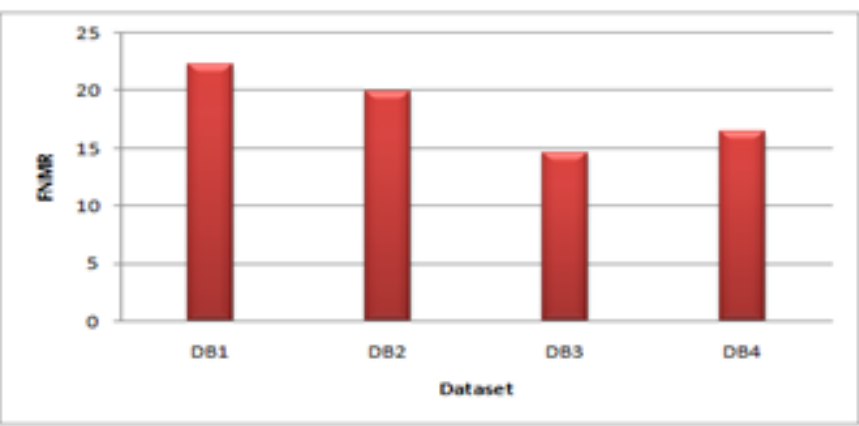

Figure 10: Column chart of the FNMR values for the four datasets

The trend of increase exhibited for these two metrics over the four datasets as shown in Figure 11.

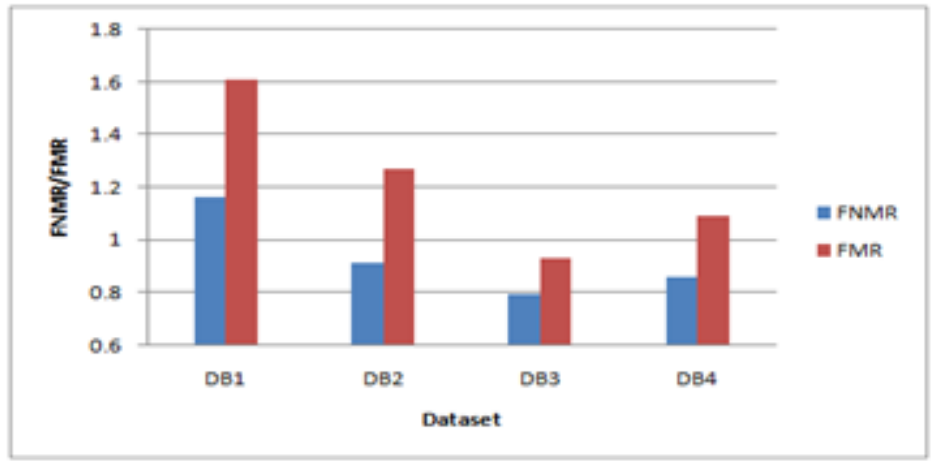

Figure 11: FNMR Matching Completion for Four Datasets

TABLE 7: FMR and FNMR for Different Algorithms

\begin{tabular}{clllllllll}
\hline \multicolumn{1}{c}{ Ref. [19] } & \multicolumn{2}{c}{ Ref. [20] } & \multicolumn{2}{c}{ Ref. [21] } & \multicolumn{2}{c}{ Current Study } \\
Set & FNMR & FMR & FNMR & FMR & FNMR & FMR & FNMR & FMR \\
\hline DB1 & 52.58 & 0 & 89.3 & 1.7 & 23.07 & 0 & 22.23 & 0 \\
DB2 & 50.03 & 0 & 88.6 & 3.7 & 19.91 & 0 & 19.85 & 0 \\
DB3 & 73.75 & 0 & 91.2 & 2.4 & 16.68 & 0 & 14.51 & 0 \\
DB4 & 65.24 & .015 & 81.3 & 0.9 & 17.09 & 0.01 & 16.47 & 0 \\
\hline
\end{tabular}


Dataset DB3 has the lowest FNMR average matching time of 0.79 seconds and FMR average matching time of 0.93 seconds followed by DB4, DB2 and DB1 with average FNMR: FMR matching time of 0.86:0.89, 0.91:1.27 and 1.16:1.61 seconds respectively. The lowest average matching rate for dataset DB3 implies that the dataset has fewest numbers of minutiae points and consequently, smallest number of computations. Similarly, the highest average matching times recorded for dataset DB1 indicate the availability of highest number of both true and false minutiae points in the images and consequently, the highest number of computations. Table 7 presents the FNMR and FMR values for four different algorithms using the same dataset (FVC2002 fingerprint database). In Table 7, the original values obtained by the authors in [19, 20] are presented. However, we implemented the algorithm proposed in [21] under the conditions of experiments in this research to obtain the stated values. The superior performance of the proposed algorithm over the other algorithms is clearly exhibited with its lowest FNMR values for all the datasets. In addition, it is the only algorithm with an FMR value of zero for all the datasets. The column charts of Figures 12 and 13 presents the graphical evidences of the superior performances of the proposed algorithm based on the values presented in Table 7.

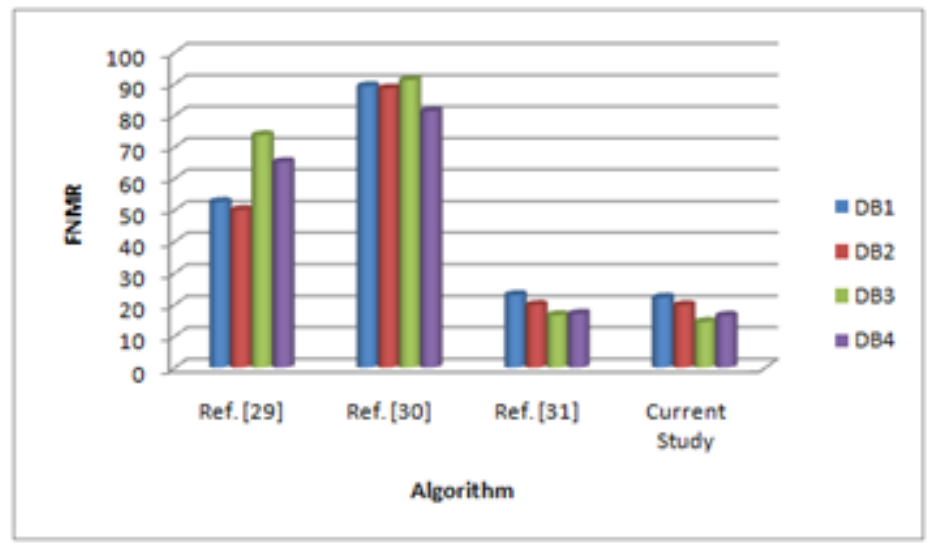

Figure 12: Colum Chart of FNMR for Four Algorithms

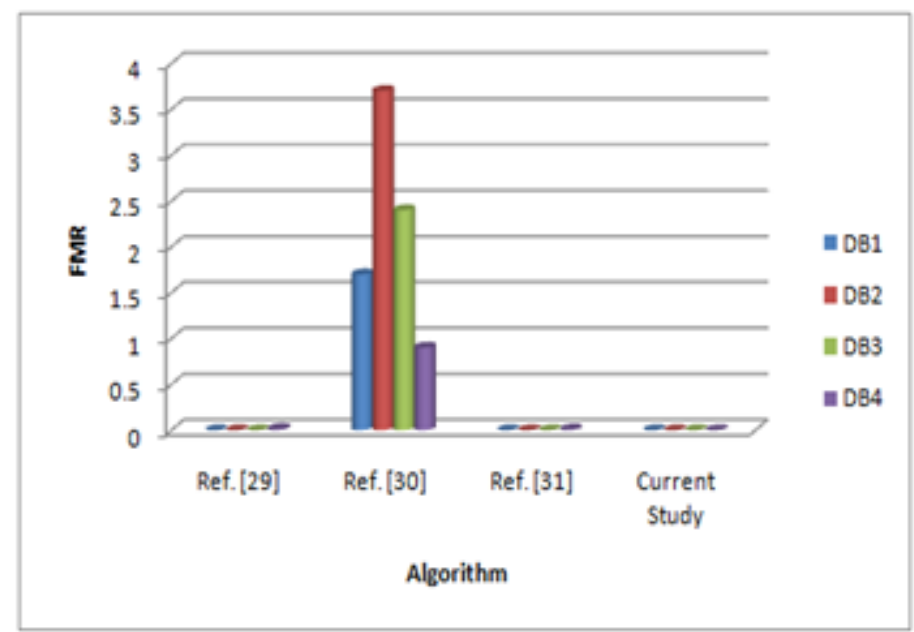

Figure 13: Colum Chart of FMR for Four Algorithms

Table 8 presents the obtained FNMR and FMR computations time in [20,21] and the current study. We also implemented the original algorithm proposed in [21] under the conditions of 
experiments in this research to obtain the stated values. For all the datasets, the proposed algorithm exhibited lower computation time, which confirms its superiority. A graphical representation of this is presented in the column charts of Figures 14 and 15 derived from values presented in Table 8.

TABLE 8: Matching Time for Different Algorithms

\begin{tabular}{ccccccc}
\hline \multicolumn{3}{c}{ Ref. [20] } & \multicolumn{2}{c}{ Ref. [21] } & \multicolumn{2}{c}{ Current Study } \\
Dataset & FNMR & FMR & FNMR & FMR & FNMR & FMR \\
\hline DB1 & 2 & 1.7 & 1.31 & 1.84 & 1.16 & 1.61 \\
DB2 & 4 & 3.7 & 1.04 & 1.32 & 0.91 & 1.27 \\
DB3 & 2 & 2.4 & 1.01 & 1.39 & 0.79 & 0.93 \\
DB4 & 3 & 0.9 & 0.91 & 1.23 & 0.86 & 0.89 \\
\hline
\end{tabular}

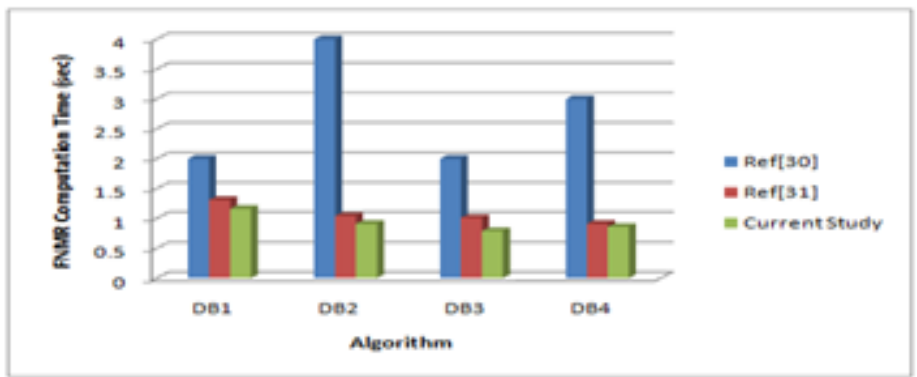

Figure 14: Colum Chart of Computation Time for Algorithms

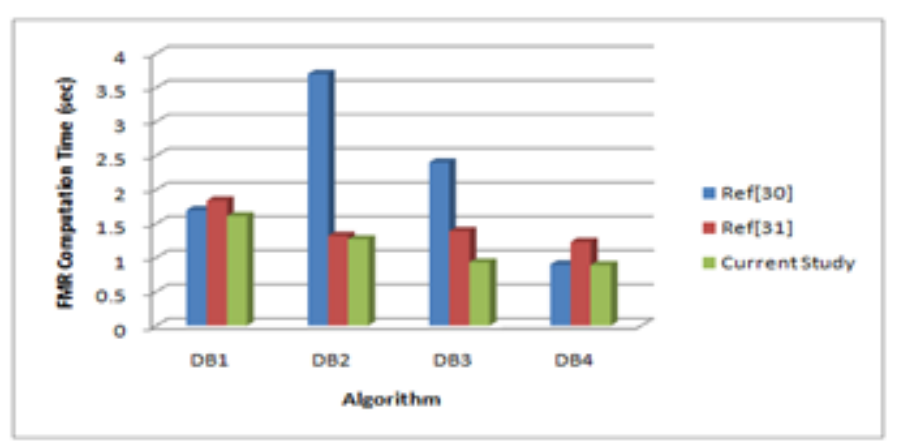

Figure 15: Colum Chart of Computation Time for FMR

Figure 16 shows the column chart of the average FNMR for four different algorithms over the four datasets. The average values are based on the data presented in Tables 7. Similarly, Figure 17 represents the column chart of the average FNMR and FMR computation times for three algorithms. These values are obtained from the data presented in Table 8. These two Figures buttressed superior performance for the proposed algorithm. 


\section{CONCLUSION}

The implementation of a proposed fingerprint pattern matching algorithm has been presented. The algorithm used the relative distances between the minutiae and the core points. This algorithm hinged on the premise that irrespective of image orientation, the minutiae points maintain constant distances with the core point for a given image size.

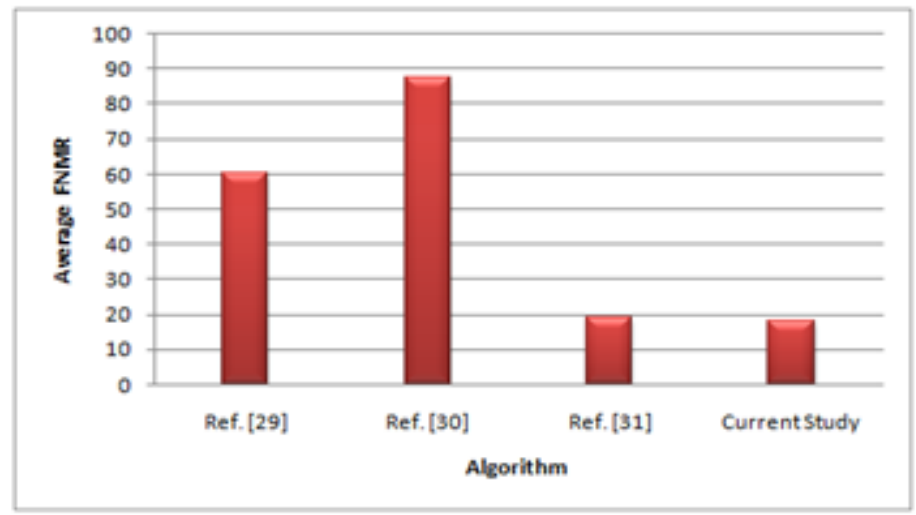

Figure 16: Colum Chart of Average FNMR for Algorithms

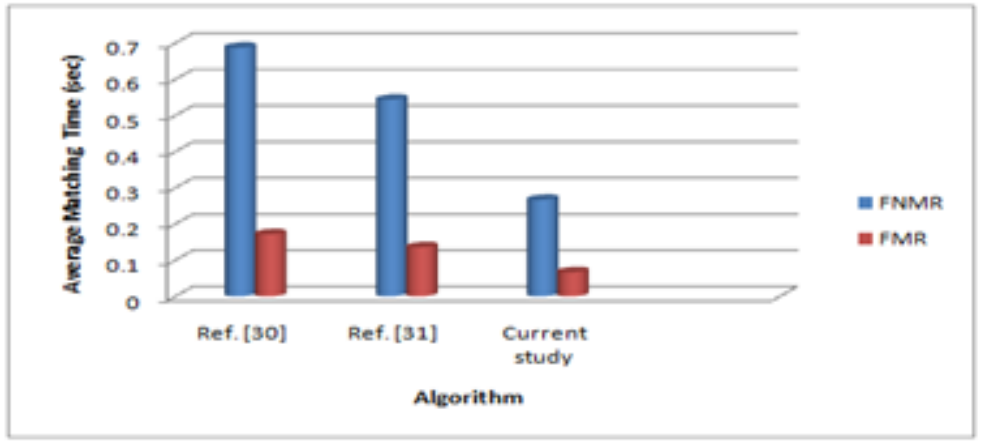

Figure 17: Colum Chart of Average Computation Time

The results obtained showed the effectiveness of the algorithm in distinguishing fingerprints from different sources with average FMR of $0 \%$. However, the ability to match images from same source depends on the qualities of such images. Since the corruption levels vary across the used datasets, the algorithm yielded different FNMR values. The first dataset is mostly affected with FNMR values of $22.23 \%$ while the third dataset is least affected with FNMR value of $14.51 \%$. The same order of performance was recorded for the FNMR and the average matching time over the datasets. There is zero tolerance of the algorithm with regard to false minutiae. A comparative review of the obtained FNMR, FMR and the computation time values with what obtained for some recently formulated algorithms over the same datasets revealed best performance for the proposed algorithm. Future research direction aims at the optimization of the proposed algorithm for further reduction in the FNMR values and the computation times. Future research direction aims, on short term, at the optimization of the proposed algorithm for increased performance. On long term, future research aims at the development of an integrated interoperable platform for the unique human identification. The specific objectives are: 
a. Development of a multi-modal biometric system for the unique identification and authentication of the individuals in Nigeria.

b. Development of a benchmark national multi-modal biometric identity database with online and real-time connection with sectoral biometric identity databases.

c. Development of a web application for hosting national biometric identification database.

d. Development of application software for unique identification and authentication of individuals.

\section{REFERENCES}

[1] Roberts C. (2005): 'Biometrics' (http://www.ccip.govt.nz/newsroom/informoationnotes/2005/biometrics.pdf)

[2] Raymond T. (2003): 'Fingerprint Image Enhancement and Minutiae Extraction', PhD Thesis Submitted to School of Computer Science and Software Engineering, University of Western Australia, pp21-56.

[3] Hong L., Wau Y. and Anil J. (2006): 'Fingerprint image enhancement: Algorithm and performance evaluation'; Pattern Recognition and Image Processing Laboratory, Department of Computer Science, Michigan State University, pp1-30

[4] Iwasokun G. B., Akinyokun O. C., Alese B. K. \&Olabode O. (2011a): 'Adaptive and Faster Approach to Fingerprint Minutiae Extraction and Validation'. International Journal of Computer Science and Security, Malaysia, Volume 5 Issue 4, page 414-424.

[5] Iwasokun G. B., Akinyokun O. C., Alese B. K. \&Olabode O. (2011b):' A Modified Approach to Crossing Number and Post-Processing Algorithms for Fingerprint Minutiae Extraction and Validation'. IMS Manthan International Journal of Computer Science and Technology, Indian, Volume 6 Issue 1, pp1-9

[6] Shenglin Y. and Ingrid M. V. (2003); A Secure Fingerprint Matching Technique, available online at www.cosic.esat.kuleuban.be/publications/article-723.pdf.Accessed 23/01/2012

[7] Giuseppe P.E and Albert N. (2003): Fingerprint Matching Using Minutiae Triangulation. Available online at http://idisk.mac.com/geppy.parziale/Public/Papers/delaunay.pdf. Accessed 23/01/2012

[8] Xinjian C., Jie T., Xin Y. and Yangyang Z. (2006): An Algorithm for Distorted Fingerprint Matching Based on Local Triangle Feature Set, IEEE TRANSACTIONS ON INFORMATION FORENSICS AND SECURITY, VOL. 1, NO. 2, page 169-177

[9] Anil K. J., Jianjiang F and Karthik N. (2010): Fingerprint Matching, IEEE Computer Society, page 36-44

[10] Zhang Weiwei, Wang Sen and Wang Yangsheng (2004): 'Pattern recognition and matching algorithm of fingerprint based on core point', National Laboratory of Pattern Recognition, Institute of Automation, Chinese Academy of Science, Beijing

[11] Shahram M. and Ali F. (2009): 'A Matching Algorithm of Minutiae for Real Time Fingerprint Identification System', World Academy of Science, Engineering and Technology 60, pp555-559

[12] Ali S. M. and Al-Zewary M. S. (1997): 'A new fast automatic technique for fingerprints recognition and identification', Journal of Islamic Academy of Sciences 10:2, 55-60.

[13] Espinosa Virginia (2002): 'A minutiae detection algorithm for fingerprint pattern recognition', IEEE Systems Magazine, pp 1-7

[14] Tsai-Yang Jea, and Govindaraju Venu (2006): 'A minutia-based partial fingerprint recognition system'. Pattern Recognition. Vol. 38, 10, pp. 1672-1684.

[15] Iwasokun G. B., Akinyokun O. C., Alese B. K. \&Olabode O. (2012): 'Fingerprint Image Enhancement: Segmentation to Thinning', International Journal of Advanced Computer Science and Applications (IJACSA), Indian, Vol. 3, No. 1, 2012

[16] Perez-Diaz A. J. and Arronte-Lopez I. C. (2010): Fingerprint Matching and Non-Matching Analysis for Different Tolerance Rotation Degrees in Commercial Matching Algorithms, Journal of Applied Research and Technology, Vol. 8 No. 2, page 186-199

[17] López A. C., Ricardo R. L., Queeman R. C. (2002): 'Fingerprint Pattern Recognition', PhD Thesis, Electrical Engineering Department, Polytechnic University, Mexico. 
[18] Maio D., Maltoni D., Cappelli R.Wayman J. L. and A. K. Jain, "FVC2002: Second Fingerprint Verification Competition," in 16th International Conference on Pattern Recognition, 2002, 2002, pp. $811-814$.

[19] Perez-Diaz A. J. and Arronte-Lopez I. C. (2010): Fingerprint Matching and Non-Matching Analysis for Different Tolerance Rotation Degrees in Commercial Matching Algorithms, Journal of Applied Research and Technology, Vol. 8 No. 2, page 186-199

[20] Peer P.(2010): 'Fingerprint-Based Verification System A Research Prototype', IWSSIP 2010 - 17th International Conference on Systems, Signals and Image Processing, Pages 150-153

[21] Li T., Liang C., and Sei-ichiro K.(2009): 'Fingerprint Matching Using Dual Hilbert Scans', SITIS, pages 553-559. 